\title{
Multi-walled carbon nanotube-coated cotton fabric for possible energy storage devices
}

\author{
S P BHARATH ${ }^{1}$, J MANJANNA ${ }^{2, *}$, A JAVEED ${ }^{1}$ and S YALLAPPA ${ }^{\mathbf{1}}$ \\ ${ }^{1}$ Department of Industrial Chemistry, Nanoscience and Technology Laboratory, Kuvempu University, Shankaraghatta \\ 577 451, Karnataka, India \\ ${ }^{2}$ Department of Chemistry, Rani Channamma University, Belagavi 591 156, Karnataka, India
}

MS received 22 January 2014; revised 14 June 2014

\begin{abstract}
A conducting cotton fabric with a resistance of $<1.5 \mathrm{k} \Omega \mathrm{cm}^{-2}$ was obtained by dip coating of multiwalled carbon nanotubes (MWCNTs) dispersed in a surfactant, sodium dodecyl sulphate (SDS). The dip coating was repeated up to 20 times to increase the loading of MWCNT as observed from optical absorption spectra $\left(\lambda_{\max }=442 \mathrm{~nm}\right)$. The field emission scanning electron microscopy (FE-SEM) image of coated fabric at different magnifications shows micro-fibril structure. Energy-dispersive X-ray analysis (EDXA) spectra show peaks for carbon and other constituent elements of SDS, Na and S. In order to improve the functionality of loaded MWCNT, the coated fabric was treated with $5 \% \mathrm{HNO}_{3}$ for $3 \mathrm{~h}$. For such a sample, the resistance decreased significantly to $1.5 \mathrm{k} \Omega \mathrm{cm}^{-2}$, whereas it is 2.0 and $2.5 \mathrm{k} \Omega \mathrm{cm}^{-2}$ for untreated and KOH-treated sample. This is in corroboration with $I-V$ characteristics, and is attributed to increased loading of MWCNT through hydrogen bonding with glycosidic group present in cotton (cellulose) fibres. The series capacitance of the MWCNT-coated fabric is about $40 \mu \mathrm{F} \mathrm{cm}^{-2}$, which is found to decrease with the increase in frequency, close to zero at about $20 \mathrm{kHz}$. A capacitor formed by placing two MWCNT-coated fabrics between etched PCB plates (terminal contacts) shows the charging capacity of about $1 \mathrm{~F}$.
\end{abstract}

Keywords. MWCNT; cotton fabrics; coating; energy storage devices; capacitance.

\section{Introduction}

Smart fabrics, also known as electronic fabrics have several built-in functions such as sensing, data processing, actuation, storage (energy or data) and communication. ${ }^{1-3}$ The potential applications of these materials include military garment devices, personal electronics, biomedical/antimicrobial textiles, etc. ${ }^{1-4}$ Conductive metal wires and other rigid fibres have been used in the past to demonstrate proofs of concept for wearable electronics. ${ }^{4}$ The coating/deposition of conductive polymers on fabrics have been carried out to develop wearable sensors. ${ }^{5}$ However, these approaches are not providing environmental compatibility, flexibility and durability. Furthermore, the manufacturing process for these fabrics is also quite complex. ${ }^{6}$

In recent times, fabrics combined with nanoscale materials have shown great interest for their potential applications viz., wearable displays, ${ }^{7}$ bio-monitoring ${ }^{8}$ and energy storage devices. ${ }^{9}$ Functionalization or modification of conventional fabrics with nanomaterials, i.e., the so-called 'smart fabrics' can be designed for improved properties ${ }^{10}$ in the development of textiles for self-cleaning, healthcare, sports, military applications and fashion. Also, incorporation of nanomaterials is helpful for enhancing the physical properties of textiles in areas such as antibacterial properties, water repellence, soil resistance, antistatic, anti-infrared, flame retardant properties

*Author for correspondence (jmanjanna@ rediffmail.com) and sustaining the textile's flexibility and strength. ${ }^{7,10}$ The MWCNT is one of the most versatile nanomaterials due to its superior material properties and responsiveness to a wide variety of environmental stimuli including changes in the temperature, $\mathrm{pH}$ and bio-chemical or mechanical stress. ${ }^{11,12}$ However, there are few reports on the treatment of fabrics materials with MWCNT through common finishing procedures. ${ }^{13,14}$ In fact, incorporating the flexible transducers/ electronics in the real system application remains the most challenging issue. Therefore, in this study, we have developed a simple method to coat cotton fabric with MWCNT by the dip-in technique. We have measured typical electronic, electrochemical and UV-blocking properties for this flexible cotton fabric to demonstrate their possible application in the real systems. The fabrication process adopted here can be easily scaled up with the roll-to-roll technique using a solution-based coating/deposition process.

\section{Experimental}

A plain cotton fabric was purchased from Hi-media Laboratory in Bangalore, India. MWCNT synthesized by the chemical vapor deposition method (Adnano Technologies Private Ltd., Bangalore, India) were used as received without further purification. Sodium dodecyl sulphate (SDS), potassium hydroxide $(\mathrm{KOH})$ and ethanol were purchased from Merck Laboratory, Bangalore, India. 


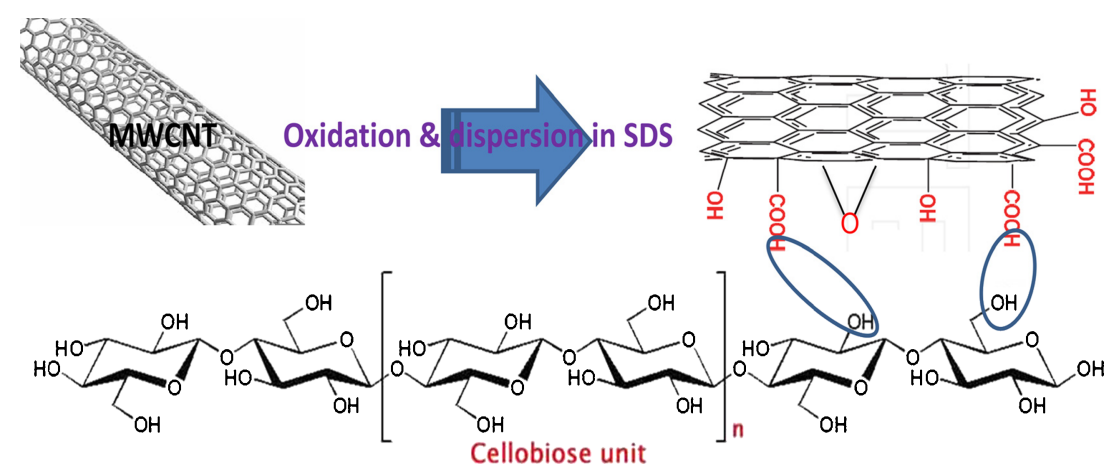

Scheme 1. Interaction of MWCNTs with cellulose/cotton fabric structure through glycosidic linkages.

MWCNT ink was prepared by dispersing MWCNT in deionized water $(1.2 \% \mathrm{wt} / \mathrm{vol})$ containing $0.1 \%\left(3.5 \mathrm{~mol}^{-1}\right)$ of SDS surfactant. It was sonicated for about $15 \mathrm{~min}$ to produce stable and clear dispersion. A clean cotton fabric $\left(1 \times 1 \mathrm{~cm}^{2}\right.$ and $\sim 1 \mathrm{~mm}$ thick) was dipped into ethanol and sonicated for $15 \mathrm{~min}$ to remove organics and detergent. It was washed with distilled water and kept for drying at $100^{\circ} \mathrm{C}$. Then it was dipped in MWCNT ink with each dip lasting for $2 \mathrm{~min}$ followed by drying at $100^{\circ} \mathrm{C}$ for $10 \mathrm{~min}$. The loading of MWCNT was increased by repeated dip and drying process. Finally, the sample was washed with deionized water to remove the excess of surfactant and dried. For comparison, MWCNT-loaded sample were treated in alkali $(5 \% \mathrm{KOH})$ and acid $\left(5 \% \mathrm{HNO}_{3}\right)$ media for $3 \mathrm{~h}$ to impart functionalization capacity to MWCNTs for effective binding on fabrics.

The series capacitance of the MWCNT-loaded fabric was measured by using precision LCR metre (SM6019) and the electrical resistance by using standard digital multi-meter. The electrical current, i.e., $I$ (current) $-V$ (voltage) characteristics of the typical samples were measured by using voltage-current meter (Keithley-6430). The adsorption of MWCNT on cotton fabrics was monitored by recording UV-vis absorption spectra (Shimadzu, 1650-PC) ranging from 200 to $800 \mathrm{~nm}$. Furthermore, the morphologies of pristine cotton fabrics and MWCNT-coated fabrics were recorded using field-emission scanning electron microscopy (FE-SEM, FEI Nova nano 600, The Netherlands). The elemental composition was determined by energy-dispersive X-ray (EDX) analysis.

\section{Results and discussion}

MWCNTs enter easily into the interwoven structure of cotton fabric due to its hydrophilic nature. Cellulose is un-branched straight chain polymer derived from D-glucose units, which condense through glycosidic bonds and forms crystalline domains. The multiple hydroxyl groups on the glucose from one chain form hydrogen bonds with oxygen atoms on the same or on a neighbour chain, holding the chain firmly together side by side and forming interwoven multiple

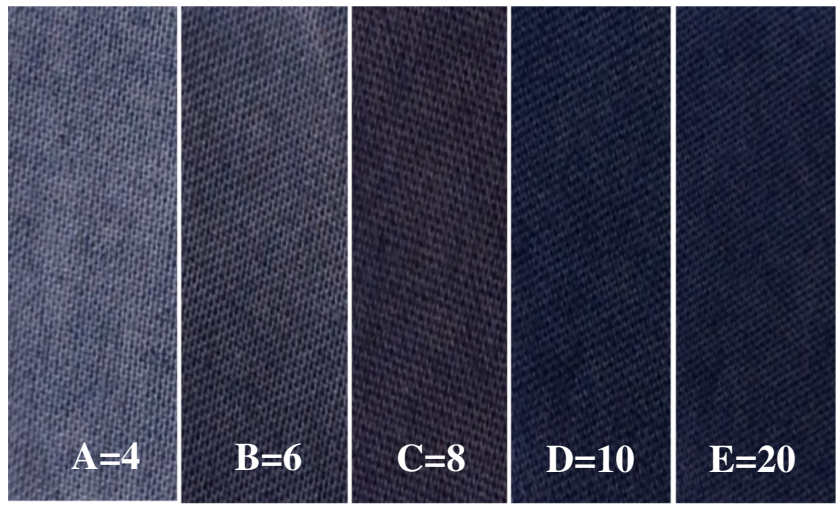

Figure 1. Unmagnified photographs of the MWCNT-coated cotton fabric (A, B, C, D and E indicate the 4, 6, 8, 10 and 20 time dipped fabric)—showing increased colour intensity with coatings.

micro-fibril structure. ${ }^{15}$ When the MWCNTs are treated with conc. $\mathrm{HNO}_{3}$ (oxidizing agent), the functional groups such as $-\mathrm{COOH}$ (carboxylic acid), -OH (hydroxyl) and -O- (epoxide) are generated through $s p^{2}$ to $s p^{3}$ hybridization of $\mathrm{C}$ atom (which are generally referred as defect sites on hexagonal graphene sheet). These functional groups facilitate in conjugating with cellulose (cotton fabric) structure through glycosidic linkages. This can be schematically represented as shown in scheme 1.

This structure allows the fibers to absorb large amounts of water or other polar solvents. Cellulose that makes up cotton is naturally insulting. In order to make a conductive fiber, we have coated MWCNTs on cotton fabric. There exists of large Van-der walls force of interaction between MWCNTs and polymers. ${ }^{16}$ Thus, we expect a similar interaction of MWCNT and cellulose fibre here. In fact, MWCNT can also form hydrogen bonds with hydroxyl functional groups present on cellulose. ${ }^{17}$ Thus, MWCNT irreversibly wraps around cellulose fibres to create a three-dimensional porous structure. MWCNT stabilized in surfactant solution seamlessly cover the exterior of every strand of cotton yarn. The coated MWCNT form the interconnected network on surface of cellulose fibres. As a result, numerous electrical 


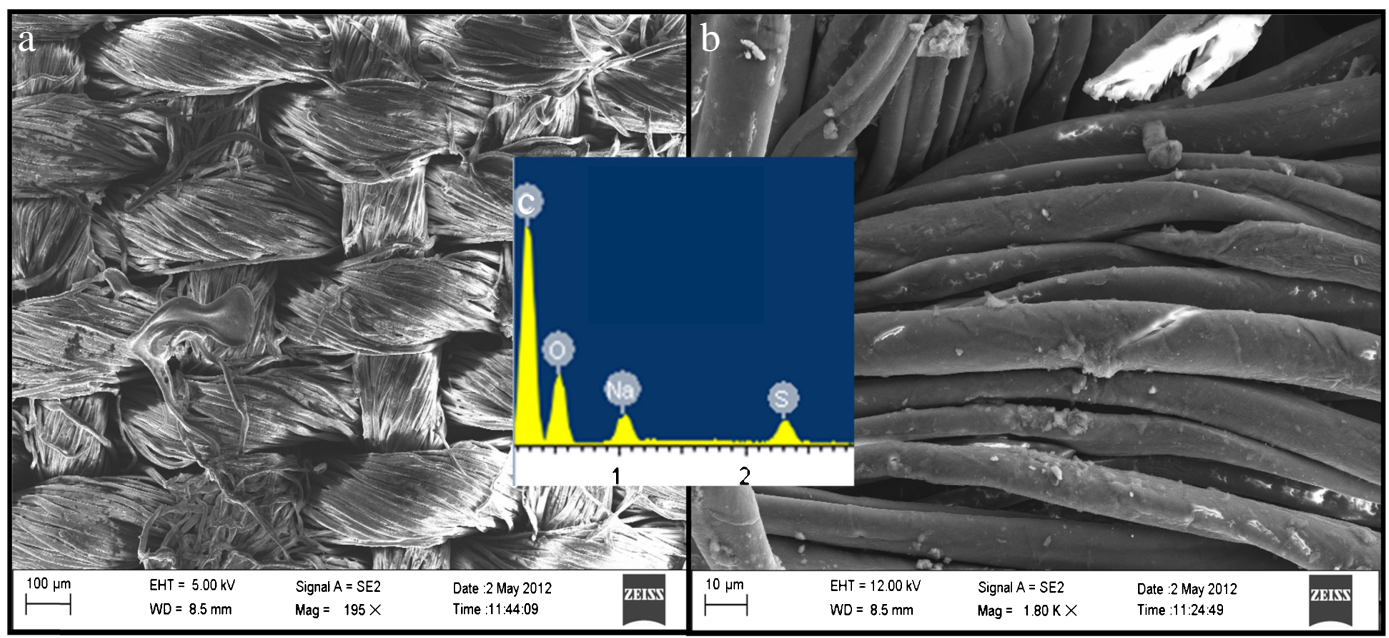

Figure 2. FE-SEM images and EDXA spectra of MWCNT-coated cotton fabric.

carrier paths can be formed. Uniformity of nanotube distribution strongly affects conductivity of cotton fabric. The colour of conductive cloths become darker with the increase in number of dipping cycle as shown in figure 1. The dip coating was repeated up to 20 times to increase the loading of MWCNTs and reaches the minimum resistance of $3 \mathrm{k} \Omega$. To avoid the electrochemical activities surfactant is completely removed by repeated distilled water wash. ${ }^{18}$

The FE-SEM image of MWCNT-coated fabric at different magnifications (figure 2) shows microfibril structure. After coating, MWCNT macro-porous structure of cotton fabric is well preserved in figure 2a. Figure 2 shows the MWCNT wrapped three-dimensional interwoven, smooth surface of cellulose fibre. The conformal coating is a result of mechanical flexibility of the individual CNT and the strong binding energy between CNT and cotton fabrics/fibres,${ }^{19}$ accounting also for the high electrical conductivity of the fabric. The previous report ${ }^{20}$ has shown that CNT films possess micro-scale porosity, which is required to maximize the specific capacitance. The micro-porous structure on top of the macro-porous fabrics leads to a 'duplex porous structure' that facilitates the easy access of electrolyte ions to the MWCNT, which is essential for high power supercapacitor applications. The EDX analysis was performed to know the percentage of the element present in the sample. The inset of figure 2 shows the EDX spectra of elemental composition, showing peaks for carbon and other residual elements such as $\mathrm{Na}$ and $\mathrm{S}$ of SDS.

The MWCNT loading was observed by optical absorption spectra (figure 3) shows the decrease in resistance of cotton for each dipping. The absorption increases rapidly up to 5 dipping. Before and after repeated water wash, optical absorption intensity remained constant as shown in figure 3 which confirms the strong adsorption of MWCNT on cellulose fibers. The increasing absorption at $442 \mathrm{~nm}$ with dipping cycles is ascribed to the formation of glycosidic linkages between the functional groups of MWCNT and cellulose. It was observed that the weight change with each dipping cycle

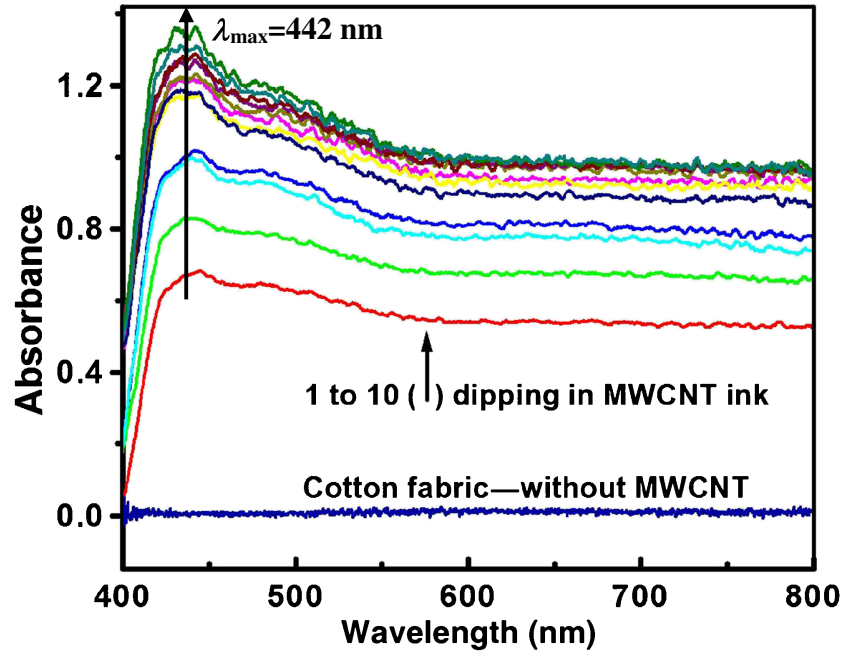

Figure 3. Optical absorption spectra of MWCNT-coated cotton fabric observed at $442 \mathrm{~nm}$ due to glycosidic linkages between MWCNT and cellulose.

was negligibly small. Hence such a mass or volume change in cotton fabric of $(1 \times 1) \mathrm{cm} \times 1 \mathrm{~mm}$ thick could not be recorded here, although it was an important parameter. In order to improve functionality of loaded MWCNT, the coated fabric was treated with $5 \% \mathrm{HNO}_{3}$ for $3 \mathrm{~h}$. For such a sample, the resistance decreased significantly to $1.5 \mathrm{k} \Omega \mathrm{cm}^{-2}$ whereas it was 2.0 and $2.5 \mathrm{k} \Omega \mathrm{cm}^{-2}$ for untreated and $\mathrm{KOH}$-treated sample. This is supported with $I-V$ characteristic curve as shown in the inset of figure 4. This is attributed to increased bonding of MWCNT through hydrogen bonding with glycosidic group present in cellulose fibres. The contact resistance could not be measured here as it was very high. The annealing in oxidizing or reducing atmosphere is expected to bring down the final value of $1.5 \mathrm{k} \Omega \mathrm{cm}^{-2}$. Nevertheless, based on this initial observation, we plan to take up detailed electrical studies of MWCNT-coated fabrics. 


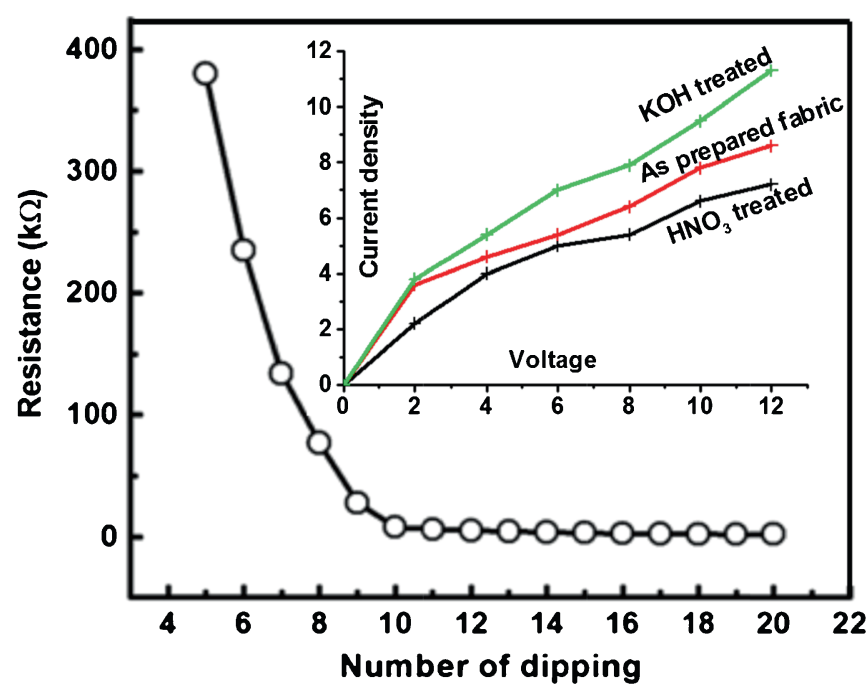

Figure 4. Resistance vs. loading of MWCNT on cotton fabric. Inset: $I-V$ characteristics of MWCNT-coated fabric with and without $\mathrm{HNO}_{3}$ or $\mathrm{KOH}$ treatment.

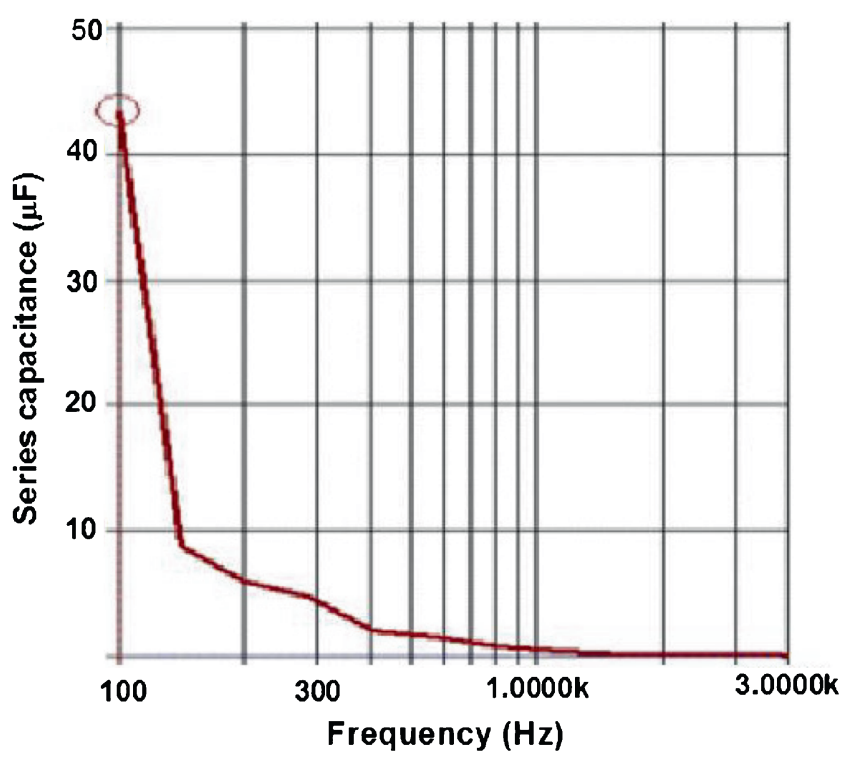

Figure 5. Capacitance of MWCNT-coated cotton fabric vs. frequency.

A previous study ${ }^{21}$ confirms micro-scale porosity of MWCNT films, which is required to raise the specific capacitance. The micro-porous structure on the top of interwoven fabric leads to increased surface area. ${ }^{22}$ The series capacitance as a function of frequency (figure 5) of the MWCNT-coated fabric is about $40 \mu \mathrm{F} \mathrm{cm} \mathrm{cm}^{-2}$ at $100 \mathrm{kHz}$. The capacitance was found to decrease with the increase in frequency, and reached close to zero at about $20 \mathrm{kHz}$. Furthermore, we have demonstrated the working capacitor by placing two coated fabrics (which are separated by a thin layer of paper as dielectric medium) between etched PCB plates (for terminal contacts). The charging capacity of this assembly was about $1 \mathrm{~F}$.

\section{Conclusions}

In this study, we have coated MWCNT on rough surface of cotton fabrics by simple dip and dry technique. The coated MWCNT formed interconnected network on cotton fibres and enhanced the electronic properties. These flexible fabrics showed resistance of $2 \mathrm{k} \Omega$ and capacitance of $40 \mu \mathrm{F}$. The resistance of MWCNT-coated cotton fabric can be easily tuned by controlling the amount of MWCNT coating and chemical (acid/base) treatment. We believe that such lightweight, flexible cotton fabrics realized by environmental friendly and low-cost methods could be used in embedded health care and wearable electronic systems.

\section{References}

1. Seymour S 2008 Fashionable technology: the intersection of design, fashion, science, and technology (Wien, New York: Springer)

2. Cherenack K, Zysset C, Kinkeldei T, Munzenrieder N and Troster G 2010 Adv. Mater. 225178

3. Scott R A 2005 Textiles for protection (Cambridge: Wood Head Publishing)

4. Gould P 2003 Mater. Today 638

5. Bowman D and Mattes B R 2005 Synth. Met. 15429

6. Pasche S, Angeloni S, Ischer R, Liley M, Lupranoe J and Voirinf G 2008 Adv. Sci. Technol. 5780

7. Gniotek K and Krucinska I 2004 Fibres Text. East. Eur. 1213

8. Lukowicz P, Kirstein T and Oster G T 2004 Methods Inf. Med. 43232

9. Park S and Jayaraman S 2003 Mater. Res. Bull. 28585

10. Karst D and Yang Y Q 2006 AATCC Rev. 644

11. Baughman R H, Zakhidov A A and Heer W A 2002 Science 297787

12. Kong J, Franklin N R, Zhou C, Chapline M G, Peng S, Cho K and Dai H 2000 Science 287622

13. Liu Y Y, Tang J, Wang R H, Lu H F, Li L, Kong Y Y, Qi K H and Xin J H 2007 J. Mater. Chem. 171701

14. Liu Y Y, Tang J and Xin J H 2004 Chem. Commun. 22828

15. Pasta M, Mantia F L, Hu L, Deshazer H D and Cui Y 2010 Nano Res. 3452

16. Zhou R, Meng C, Zhu F, Li Q, Liu C, Fan S and Jiang K 2010 Nanotechnology 21345701

17. Kang T J, Choi A, Kim D, Jin K, Seo D K, Jeong D H, Hong S, Park Y W and Kim Y 2011 Smart Mater. Struct. 20 015004

18. Shim B S, Chen W, Doty C, Xu C and Kotov N A 2008 Nano Lett. 84151

19. Hertel T, Walkup R E and Avouris P 1998 Phys. Rev. B 58 13870

20. An K H, Kim W S, Park Y S, Choi Y C, Lee S M, Chung D C, Bae D J, Lim S C and Lee Y H 2001 Adv. Mater. 13497

21. Hu L, Pasta M, Mantia F L, Cui L, Deshazer H D, Choi J W, Han S M and Cui Y 2010 Nano Lett. 10708

22. Hummers W S and Offerman R E 1958 Am. J. Chem. Soc. 80 1339 